\title{
Effects of Mindfulness-Acceptance-Commitment (MAC) on Sport-Specific Dispositional Mindfulness, Emotion Regulation, and Self-Rated Athletic Performance in a Multiple-Sport Population: an RCT Study
}

\author{
Torbjörn Josefsson ${ }^{1} \cdot$ Andreas Ivarsson ${ }^{1} \cdot$ Henrik Gustafsson ${ }^{2} \cdot$ Andreas Stenling $^{3} \cdot$ Magnus Lindwall $^{4}$. \\ Rasmus Tornberg ${ }^{1} \cdot$ Jan Böröy ${ }^{1}$
}

Published online: 13 February 2019

(C) The Author(s) 2019

\begin{abstract}
Objectives The aim of the study was to examine mediating effects of emotion regulation and sport-specific dispositional mindfulness on self-rated athletic training performance, following the Mindfulness-Acceptance-Commitment (MAC) intervention, compared to a Psychological Skills Training (PST) control group.

Methods Sixty-nine competitive elite athletes who did not have any prior experience with mindfulness- and acceptance-based exercises, were recruited and randomly assigned into either a MAC group or a traditional PST group. Latent growth curve analyses were performed to examine longitudinal relationships among the study variables. Mediation analyses were conducted to test if the growth trajectory of each of the proposed mediators mediated the relationship between the intervention and perceived performance (measured at T3).

Results Findings showed that the MAC intervention had an indirect effect on self-rated athletic training performance through changes in dispositional mindfulness and emotion regulation respectively. Further, the MAC-group obtained greater post-test improvements in athletic mindfulness, emotion regulation abilities, and perceived performance compared to the PST group.

Conclusions Overall, findings suggest that dispositional athletic mindfulness and emotion regulation may function as important mechanisms in MAC, and that the MAC approach is a more effective intervention compared to the PST condition in reducing emotion regulation difficulties, as well as enhancing sport-relevant mindfulness skills and perceived athletic training performance in elite sport.
\end{abstract}

Keywords Emotion regulation $\cdot$ MAC $\cdot$ Mediation analysis $\cdot$ Mindfulness $\cdot$ Mindfulness-acceptance-commitment . Performance $\cdot$ PST

In competitive sport, the development of psychological interventions designed to optimize athletic performance has gained considerable interest since the early 1970s. Most of these interventions have been based on traditional psychological skills

Torbjörn Josefsson torbjorn.josefsson@hh.se

1 Center of Research on Welfare, Health, and Sport, Halmstad University, Kristian IV:s väg 3, 30118 Halmstad, Sweden

2 Faculty of Health, Science, and Technology, Karlstad University, Universitetsgatan 2, 65188 Karlstad, Sweden

3 Department of Psychology, Umeå University, 90187 Umeå, Sweden

4 Department of Psychology, University of Gothenburg, Läroverksgatan 5, 40530 Gothenburg, Sweden training (PST), mainly grounded in cognitive techniques and principles (Gustafsson et al. 2017). PST has been applied to develop increased self-control over internal processes that may inhibit sport performance (e.g., dysfunctional thinking and negative affective responses) by employing various techniques, for example thought-stopping and arousal control.

However, during the last decade, several researchers have critically discussed the effectiveness of these control strategies by pointing out that athletes still seem to experience difficulties in employing PST-related techniques (Birrer et al. 2012), and furthermore, that the empirical support for regular PST approaches, in relation to performance, is limited (Moore 2009). A vast body of research clearly indicates that striving for mental control in an attempt to get rid of unwanted emotional and mental content ends up not only being unsuccessful; it may paradoxically even be counterproductive. 
According to the theory of ironic mental processes, struggling to control or suppress a certain thought may often lead to an increasing engagement in the very thought one tries to avoid (Wegner 1989; Wenzlaff and Wegner 2000). In addition, selffocused attention on automatized movements and a deliberate attempt to control them have also been associated with decreases in sport performance (see Masters and Maxwell 2008, for an overview).

Inspired by contemporary clinical research regarding the efficacy of mindfulness- and acceptance-based interventions (MABI) (Khoury et al. 2013), Gardner and Moore (2004) introduced the Mindfulness-Acceptance-Commitment (MAC) protocol, specifically designed to enhance athletic performance. The MAC approach, based primarily on the principles of Acceptance and Commitment Therapy (ACT; Hayes et al. 1999), offers a different view of athletic performance enhancement that challenges the well-established principles of PST, frequently used by sport psychologists.

In contrast to traditional PST techniques, the MAC approach is not based on the assumption that it is necessary to create an ideal inner performance state by consistently scanning the mind in order to detect and eliminate negative internal experiences. According to the MAC approach, optimal performance may rather be enhanced by a present-centered, nonjudgmental awareness of inner and outer stimuli, where these experiences are seen as impermanent events that come and go in the ongoing stream of consciousness. In addition, a general attitude of acceptance is employed towards all mental and emotional content. The MAC approach also targets external attentional focus on task-relevant stimuli rather than selffocused attention on internal processes. Finally, the athlete needs to be deeply committed to his/her sport; all chosen behaviors and actions should consistently be in line with personal values and, accordingly, support athletic goals (Gardner and Moore 2007).

However, it is not only important to study effects of MABIs on athletic performance; it is just as crucial to explore the underlying mechanisms behind these effects (Gardner 2009). Indeed, a greater knowledge of which specific elements in MAC that influence changes in athletic performancerelevant outcomes will make it possible to further refine and improve the effectiveness of these interventions. According to Gardner (2009), the MAC intervention may not directly cause an effect on performance; the effect is rather hypothesized to go indirectly through another variable, the mechanism that is, which in turn results in improvements in performance-relevant outcomes.

Lindsay and Creswell (2017) recently introduced the Monitor and Acceptance Theory (MAT) where they propose that the particular combination of the two components, (i) attention monitoring, defined as "ongoing awareness of present-moment sensory and perceptual experiences" ( $\mathrm{p}$. 50), and (ii) acceptance, are the two major underlying mechanisms, responsible for MABI-related effects on psychological and physical health outcomes. Improved attention ability is hypothesized to increase awareness of ongoing stimuli: positive as well as negative ones. However, without employing acceptance skills to current experiences, increased self-focused attention to negative and/or unwanted stimuli may be counterproductive and may also enhance as well as prolong affective states, such as anxiety and worry (Lindsay and Creswell 2017). For example, attention capacity may be strengthened by attention monitoring but without acceptance, focus on current tasks may be distracted by negative thoughts and disturbing emotions, and result in performance decreases (Gardner and Moore 2012). Considering that anxiety often interferes with performance, Borkovec (2002) argues that present-centered attention on external stimuli (rather than on internal experiences) increases the possibility to accurately process new information, which in turn may provide the optimal conditions for adaptive behavioral responses to current situations. Together, attention and acceptance skills may reduce affective reactivity, improve performance on cognitive tasks in affective contexts, and reduce stress (Lindsay and Creswell 2017).

Consistent with Borkovec's (2002) view as well as the MAT approach, researchers in the sport psychology area (e.g., Gardner and Moore 2012; Marks 2008) suggest that the mechanisms of action in sport-specific MABIs may be heightened contextual awareness, better attentional control, and improved acceptance skills that may increase athletes' abilities to keep attentional focus on current sport-relevant tasks, without neglecting other potentially important information. Based mainly on neuropsychological research on meditation and cognition that show changes in brain structure and greater activity and processing in attention-related parts of the brain in experienced meditators (e.g., Brefczynski-Lewis et al. 2007; Cahn and Polich 2009; Lutz et al. 2004; Pagnoni and Cekic 2007) as well as superior performance on cognitive tasks for mindfulness meditation conditions compared to control groups (see Chiesa, Calati, and Serretti, 2010, for an overview), mindfulness training may also facilitate a more efficient and economical way of using cognitive resources (Gardner and Moore 2012; Marks 2008). As Marks (2008) put it, mindful athletes may have developed an "ability do to more with less" (p. 231).

In an attempt to explain how mindfulness works in sports and what its mechanisms are, Birrer et al. (2012) proposed a theoretical model in which mindfulness practice and/or dispositional mindfulness (trait mindfulness that usually is conceptualized as involving attention regulation, awareness, and acceptance) are hypothesized to generate a number of certain mindfulness impact mechanisms (i.e., acceptance skills, attention skills, and various interrelated mechanisms linked to emotion regulation), which in turn are assumed to promote various performance-related psychological skills (e.g., coping, recovery, motivation). Furthermore, increases in dispositional 
mindfulness are hypothesized to mediate the relation between mindfulness practice and improvements in athletic performance-relevant outcomes (Birrer et al. 2012).

Consistent with the model proposed by Birrer et al. (2012), Moore (2009) postulates that a crucial mechanism of change in the MAC program is emotion regulation, and that an important overall goal with the MAC intervention is to improve adaptive emotion regulation strategies. Emotion regulation is a complex, context-dependent process (Chambers et al. 2009) that commonly is defined as "the processes by which individuals influence which emotions they have, when they have them, and how they experience and express these emotions" (Gross 1998, p. 275), and such a process may be either "automatic or controlled, conscious or unconscious" (Gross 1998, p. 275). In line with this definition, emotion regulation has been conceptualized as a construct including five certain abilities: "(a) awareness and understanding of emotions, (b) acceptance of emotions, (c) ability to control impulsive behaviors and behave in accordance with desired goals when experiencing negative emotions, and (d) ability to use situationally appropriate emotion regulation strategies flexibly to modulate emotional responses as desired in order to meet individual goals and situational demands" (Gratz and Roemer 2004, pp. 42-43). In accordance with this view, adaptive emotion regulation is a process of controlling behavior when negative affects arise rather than trying to control or suppress unwanted affects and emotions (Gratz and Tull 2010).

In general, MABIs are expected to improve emotion regulation in several ways. First, mindfulness practice may improve attention capacity and promote a deeper awareness of current thoughts and emotions that may help individuals to quickly identify disturbing emotions that need to be regulated (Josefsson et al. 2017; Roemer et al. 2015). Second, common MABI features such as observing and verbalizing emotions are hypothesized to increase emotional awareness, which may facilitate the ability to both identify and distinguish between different emotions and affects, and hence, inhibit automatic emotional reactivity and emotional intensity (Coffey et al. 2010; Gratz and Tull 2010). Third, the non-judgmental and non-evaluative attitude taught in various MABIs may lead to both greater acceptance of unwanted emotions as well as emotional willingness to experience current emotions (Gratz and Tull 2010). Emotional acceptance and willingness are then hypothesized to improve emotion regulation abilities, decrease experiential avoidance (the tendency to handle emotions, thoughts, and all unwanted internal experiences by avoiding them), and increase tolerance of feared and avoided affective states (Gratz and Tull 2010; Hayes and Feldman 2004).

Meta-analytical results show large effect sizes on athletic performance-related variables as well as on process-oriented variables such as dispositional mindfulness in favor of heterogeneous MABIs compared to control conditions in athlete populations (Bühlmayer et al. 2017). However, only two of the included studies in Bühlmayer et al. (2017) specifically examined the MAC program (Goodman et al. 2014; Zhang et al. 2016). Zhang et al. (2016) found statistically significant betweengroup differences in dispositional mindfulness as well as in athletic performance in favor of the MAC condition. Goodman et al. (2014), who actually studied a modified five-week MAC intervention, did not examine effects on athletic performance but found a statistically significant between-group difference in dispositional mindfulness in favor of the MAC group compared to an inactive control group. Similarly, Gross et al. (2016) found that the MAC participants scored statistically significantly higher than a PST comparison group on dispositional mindfulness. On the other hand, no statistically significant differences in sport performance were found between MAC and the PST comparison group. However, a statistically significant within-group effect was found showing improvements in sport performance for the MAC participants. Furthermore, a decrease in emotion regulation difficulties between pre- and follow-up was found for the MAC group (Gross et al. 2016).

In sum, reviews (e.g., Noetel et al. 2017) and metaanalytical results (Bühlmayer et al. 2017) generally show that MABIs enhance both sport performance-relevant outcomes and process-related variables, such as dispositional mindfulness, albeit more methodologically strong empirical studies are needed to further establish the magnitude these effects may have (Noetel et al. 2017). Specifically, RCTs with active control groups investigating effects of standardized MABIs on sport performance-relevant outcomes as well as potential mechanisms (i.e., dispositional mindfulness, emotion regulation) are warranted (e.g., Noetel et al. 2017).

The majority of RCTs examining multi-modal MABI-effects have used inactive controls, making it difficult to detect unique mindfulness effects (MacCoon et al. 2012). To accurately examine effects of MABIs as well as specific mindfulness mechanisms, it is not only necessary to employ an active control group that is structurally equivalent to the experiment condition. The control condition should also be potentially therapeutic in relation to athletic goals and performance, include meaningful session activities where the participants are able to discuss particular problems and issues of relevance to sport, be expected to have positive athletic outcomes, and not include mindfulness or any other MAC-specific ingredients (see Baskin et al. 2003; MacCoon et al. 2012; Mohr et al. 2009 , for more information about active control conditions).

Noetel et al. (2017) particularly highlight the need to design intervention studies where mediation models are explored because that would make it possible to study the causal nature of presumed MABI mechanisms and performance outcomes. Aside from studying the potential major mechanism in MAC, emotion regulation (Moore 2009), Birrer et al. (2012) emphasize the necessity of also exploring if changes in dispositional mindfulness mediate the relation between mindfulness practice and performance-relevant outcomes. 
Given that all published studies investigating dispositional mindfulness in a sport context, so far, have used measures that are not specifically designed for athletes, Josefsson et al. (2017) argued that it would be more appropriate to use a sport-specific mindfulness questionnaire (e.g., AMQ; Zhang et al. 2015) rather than a general mindfulness measure when studying dispositional mindfulness in athlete populations. Based on the aforementioned proposed theoretical model by Birrer et al. (2012), not yet empirically tested in an intervention trial, the main aim of the current study was to examine if changes in emotion regulation and dispositional mindfulness act as mediators in the relation between the MAC intervention (independent variable) and self-rated athletic performance (dependent variable).

\section{Method}

\section{Participants}

Sixty-nine competitive elite athletes (36 men and 33 women, mean age $=20.9$, SD $=4.17$ ), who did not have any prior experiences of mindfulness- and acceptance-based exercises, were recruited from three populations, in the southwest area of Sweden: a national league floorball club $(n=38)$, a golf section at a sport high school, $(n=23)$, and a university program specifically designed for elite athletes $(n=8)$. The participants represented one out of five sports: floorball $(n=38)$, golf ( $n=27)$, football (soccer) $(n=2)$, cycling ( $n=1)$, wrestling $(n=1)$. The competitive levels among the participants were as follows: local $(n=1)$, regional $(n=5)$, national $(n=$ 32 ), and international $(n=26)$. The participants were randomized into either a MAC group or a PST control group, by using a restricted shuffled randomization approach (Schultz and Grimes 2002). The total MAC sample (including all three samples: sport high-school, university, and floorball club) consisted of 36 participants (17 men and 19 women, mean age $=20.9, \mathrm{SD}=4.24$ ), and the number of participants in the active control group (also including participants from all three samples) was 32 (18 men and 14 women, mean age $=21.0$, $\mathrm{SD}=4.16)$. One participant in the control group was excluded from the analysis because of previous major experiences in meditation and mindfulness practice. Means and standard deviations for attendance rates (maximum seven sessions) were $5.47(\mathrm{SD}=1.21)$ for the MAC group and $5.61(\mathrm{SD}=1.50)$ for the control group.

\section{Procedure}

Letters including a presentation of the current study and an inquiry to let their athletes participate in a repeated measure study were sent by email to the sports director at a Swedish National League floorball club in the first division, the director of studies for a sport high-school, and the director of studies for the university program. All three instances were interested to take part in the study and meetings were set up where the study was presented more thoroughly. Permissions to present the study to the athletes were given from representatives (i.e., coaches, and the sports director) from all three instances. At the presentations, all athletes were given written and verbal information about the study design. They were further informed that the data would be treated confidentially, and that they had the right to withdraw from the study at any time. The first data collection was administered prior to the first session, the second data collection prior to the fourth session, and the third data collection after the last session. Thus, the interval was approximately three weeks between each data collection.

MAC Intervention In total, the MAC group had seven 50-min sessions and met once a week for seven weeks. Two MAC instructors were recruited, one delivering the intervention for the floorball team and the other for the university and sport high school students. The instructor for the golfers and the university students has a Ph.D. in psychology and has previously delivered the MAC intervention to various groups of athletes on several occasions. The instructor for the floorball team has a master's degree in psychology and has been working as a sport psychology consultant for individual- as well as team-sport athletes for more than ten years, and is also experienced in working with the MAC protocol.

The MAC program is a flexible 7-module protocol (Gardner and Moore 2007). (1) Preparing the client with psychoeducation: information about theoretical and practical aspects of the intervention and an introduction to the structure and the content of the full MAC program. (2) Introducing mindfulness and cognitive defusion: the meaning of these concepts are defined and explained, and how they can be applied in a sport context. (3) Introducing values-driven behavior: the relation between goals, values, and behaviors is presented and discussed. The differences between valuesdriven behavior versus emotion-driven behavior are emphasized. (4) Introducing acceptance: the primary purpose of this module is to develop an understanding about the consequences associated with experiential avoidance, and the potential benefits with applying experiential acceptance when striving for improved performance. (5) Enhancing commitment: presentation of the concepts motivation and commitment, and how they differ from one another, and their relation to performance-related values and behaviors. (6) Skill consolidation and poise - combining mindfulness, acceptance, and commitment: the main purpose of this module is to attain and maintain behavioral flexibility. (7) Maintaining and enhancing mindfulness, acceptance, and commitment: discussion about how to maintain and deepen the skills learned in previous sessions after the intervention has been completed. Written and oral evaluations of the program are also included in this 
module. In each session, various mindfulness exercises are included (e.g., brief centering exercise, task-focused attention exercises) (Eifert and Forsyth 2005). In addition, the participants were encouraged to do mindfulness exercises in between sessions (audio files with guided mindfulness exercises). For more detailed information about the content in each MAC module, see Gardner and Moore (2007).

Control Intervention In an effort to create a structurally equivalent control condition as closely matched to the MAC intervention as possible, a traditional PST intervention was developed for this study. Both MAC and the PST interventions were structurally equivalent in terms of number of sessions and session lengths (seven sessions, one session/week, $50 \mathrm{~min} / \mathrm{session}$ ), and having a group format. Two experienced control group instructors (Ph.Lic. and master's degree in psychology respectively), both of them experienced in working with PST methods, were employed to deliver the PST intervention.

The PST program consisted of the following topics. (1) Introduction to sport psychology: the topic sport psychology was introduced to the athletes, emphasizing how athletes usually practice and apply sport psychological features in today's sport. (2) Interpersonal relationships in sports - communication: basic skills for communication in a sport context were discussed (Harge 2006). (3) Interpersonal relationships in sports - roles and norms: the dynamic model for sport groups (Carron and Hausenblas 1998) was presented as a theoretical framework for this topic. (4) Motivation and motivational climate: theories such as self-determination theory (Deci and Ryan 2000) and achievement goal theory (Elliot et al. 2011) were introduced and discussed. (5) Goal setting: the S.M.A.R.T.S. model (Smith 1994) was presented for the athletes. (6) Self-confidence/self-efficacy in sport: the theory of self-efficacy (Bandura 1997) was presented to the athletes. (7) Evaluation and discussion about the content of the intervention: in this last session, the athletes discussed what they had learned from the different topics in the intervention and how they could be regularly implemented in a sport context.

\section{Measures}

Prior experience in meditation and meditation-related practices such as yoga and relaxation was assessed on a nominal scale (yes-no), and an open follow-up question where the participants were asked to specify type of practice (see Table 1). Performance was measured by a single item ("During the last two weeks, I rate my training performance as...") on a 10-point Likert scale (ranging from "very poor" to "very good"). Similar performance measures have been used in other studies assessing self-rated sport performance (e.g., Hasker 2010; Lane and Chappell 2001; Lowther et al. 2002). We chose to use a single-item measure to study perceived
Table 1 Descriptive statistics of previous experiences of meditationrelated practice, and the allocation of athletes in the MAC group and in the control group

\begin{tabular}{lll}
\hline & MAC & Control \\
\hline$N$ & 36 & 33 \\
Prior experience of meditation-related & $7 / 19.4$ & $11 / 33.3$ \\
$\quad$ practice $(n / \%)$ & 6 & 5 \\
Yoga $(n)$ & 2 & 5 \\
Relaxation $(n)$ & 0 & 0 \\
Meditation $(n)$ & $19 / 52.8$ & $19 / 57.6$ \\
Floorball club $(n / \%)$ & $4 / 11.1$ & $4 / 12.1$ \\
University $(n / \%)$ & $13 / 36.1$ & $10 / 30.3$ \\
Sport high school $(n / \%)$ & & \\
\hline
\end{tabular}

overall training performance mainly because a single-item indicator may generate more valid data than multiple-item measures when assessing a global concept. Furthermore, validity and reliability of single-item measures are generally similar to that of psychometric properties in equivalent multiple-items measures (see Youngblut et al. 2013, for an extended discussion).

The Athlete Mindfulness Questionnaire The AMQ (Zhang et al. 2015) is a recently developed 16-item questionnaire, using a 5 -point Likert scale $(1=$ strongly disagree to $5=$ strongly agree), designed to assess dispositional mindfulness in a sport context, and consists of three subscales: Presentmoment attention ("If I notice that my mind is wandering, I can quickly get back to focusing on my training or competition," Awareness ("When the situation changes during the competition, I am aware of the thoughts and ideas that flashed across my mind," and Acceptance ("During training and competition, it doesn't matter if my thoughts and feelings are comfortable or not, I put up with all of them"). Initial analyses indicate good validity and reliability (Zhang et al. 2015). Higher scores indicate greater dispositional athletic mindfulness. The Swedish translation of the original AMQ was done by the authors of the present study. An independent translator, blind to the original questionnaire, performed a back translation that resulted in minor changes on some of the items. Only the total AMQ scale, including all three subscales, was used in the analyses. In the present study, the reliability coefficients were $=0.84(\mathrm{~T} 1),=0.89(\mathrm{~T} 2)$, and $=0.87(\mathrm{~T} 3)$.

Difficulties in Emotion Regulation Scale The complete Difficulties in Emotion Regulation Scale (DERS; Gratz and Roemer 2004) is a 36-item questionnaire designed to assess six specific dimensions of emotional dysregulation, using a 5point Likert scale ranging from 1 (almost always) to 5 (almost never). The DERS subscale Non-acceptance of emotional responses was excluded in the analysis because acceptance has 
in factor analyses been categorized first and foremost as a mindfulness component rather than an emotion regulation feature (Coffey et al. 2010). Moreover, the subscale Clarity was also excluded because it has been suggested to be a distinct mechanism in itself, separated from emotion regulation, theoretically (Birrer et al. 2012) as well as in factor analyses (Coffey et al. 2010). Hence, four of the six subscales in the DERS were combined into a total emotion regulation scale: Difficulties engaging in goal-directed behavior ("When I'm upset, I have difficulty focusing on other things"), Impulse control difficulties ("I experience my emotions as overwhelming and out of control"), Lack of emotional awareness ("When I'm upset I take time to figure out what I'm really feeling"), Limited access to emotion regulation strategies ("When I'm upset, it takes me a long time to feel better"). Higher scores indicate greater problems with emotion regulation. Validity as well as reliability has in general been shown to be consistent across various populations (Ritschel et al. 2015). Reliability coefficients for the total four-subscale DERS in the present study are $=0.91(\mathrm{~T} 1),=0.89(\mathrm{~T} 2)$, and $=0.92(\mathrm{~T} 3)$.

\section{Data Analyses}

Descriptive statistics were calculated using JASP software package (JASP Team 2018. JASP Version 9.0). We performed linear regression analysis to examine the direct effect of the intervention on training performance. To examine change in the two proposed mediators (i.e., emotion regulation and dispositional mindfulness), unconditional linear latent growth curve models (LGCMs) were estimated. Finally, we estimated two mediation analyses to examine if the growth trajectory (i.e., the slope) of dispositional mindfulness and emotion regulation mediated the effect of the intervention on training performance (measured at T3). The intervention variable was coded $1=$ intervention and $2=$ control. For more information about this type of mediational models see, for example, Roesch et al. (2010).

We performed the regression analysis, LGCM, and mediation analyses in Mplus 8.0 (Muthén \& Muthén, 1998-2017), using the Bayesian estimator. In comparison to the more traditional frequentist framework, the Bayesian statistical framework is based on different assumptions (for more information about the differences in assumptions between these two frameworks, see, for example, Ivarsson et al. 2015; Stenling et al. 2015). One of the advantages with the Bayesian statistical framework compared to the frequentist framework is the higher likelihood of producing reliable estimates even with small sample sizes (Song and Lee 2012). Due to the less restrictive distributional assumptions, related to the Bayesian framework, the normality assumption is not needed to be fulfilled to perform the analyses (Yuan and MacKinnon 2009). The traditional normality assumption is particularly difficult to fulfill in small samples and Bayesian statistics can therefore be favorable is small samples.
For all the LGCM, we used the Markov Chain Monte Carlo simulation procedures with a Gibbs sampler. We estimated all using 200,000 iterations. A potential scale reduction factor around 1.0 was considered evidence of convergence (Kaplan and Depaoli 2012). Model fit was assessed using the posterior predictive $p(\mathrm{PP} p)$ value and its accompanying 95\% confidence interval. In Mplus, "the $95 \%$ confidence interval is produced for the difference in the $f$ statistic for the real and replicated data. A positive lower limit is in line with a low posterior predictive $p$ value and indicates poor fit" (Muthén and Asparouhov 2012, p. 315).

For all estimated paths, a credibility interval (CI) was generated. The credibility interval indicates the probability (e.g., $95 \%$ ) that the parameter of interest lies between the two values given the observed data. If the $95 \% \mathrm{CI}$ around the parameter estimate did not include zero, we considered it to be a credible parameter estimate (i.e., we could reject the null hypothesis of no effect; cf. Zyphur and Oswald 2015). In the regression analysis, a mean prior for the structural parameter was included. This prior was based on estimates from a systematic review on mindfulness and sports performance (Noetel et al. 2017). For the unconditional LGCM, the default priors in Mplus were used. Priors (i.e., means) for the structural parameter estimates in the mediation analyses were obtained from recently published research (we refer to these as empirically derived priors; for exact estimates and references, see Table 3). Using different prior specifications can potentially lead to different results; hence, we followed recommendations in the literature (e.g., Van de Schoot et al. 2014) and performed a sensitivity analysis. In the sensitivity analysis, the hypothesized models (i.e., using priors from published research on the means and moderate variance specifications; model A) were compared with two other models using the same mean parameter but with different variance priors. In the first comparison model, we used a highly informative (i.e., high precision; model B) prior for the variance, while in the second comparison model, we used a less/weakly informative prior (i.e., lower precision; model C).

\section{Results}

The descriptive results showed that both groups of athletes showed rather high levels of dispositional mindfulness, selfreported performance, and adherence. They also showed moderate to low values of emotional regulation difficulties. For more information about the descriptive results, see Table 2.

\section{Linear Regression Analysis for the Relation Between MAC and Performance}

Sensitivity analyses showed similar model fit for all three models (combined with similar DIC values). We selected the model with a highly informative prior on the variance because 
Table 2 Means and standard deviations on dispositional mindfulness, emotion regulation, performance, and adherence for all three measurement points

\begin{tabular}{|c|c|c|c|c|}
\hline & $N$ & $\begin{array}{l}\mathrm{MAC} \\
M(\mathrm{SD})\end{array}$ & $N$ & $\begin{array}{l}\text { Control } \\
M \text { (SD) }\end{array}$ \\
\hline AMQ total 1 & 33 & $3.38(0.52)$ & 31 & $3.64(0.52)$ \\
\hline AMQ total 2 & 31 & $3.50(0.53)$ & 28 & $3.87(0.53)$ \\
\hline AMQ total 3 & 30 & $3.87(0.50)$ & 25 & $3.78(0.58)$ \\
\hline DERS total 1 & 33 & $2.50(0.72)$ & 31 & $2.40(0.67)$ \\
\hline DERS total 2 & 31 & $2.43(0.69)$ & 28 & $2.33(0.58)$ \\
\hline DERS total 3 & 30 & $2.00(0.62)$ & 25 & $2.19(0.67)$ \\
\hline Performance 1 & 31 & $6.36(1.58)$ & 31 & $6.87(1.18)$ \\
\hline Performance 2 & 27 & $6.81(1.73)$ & 26 & $7.00(1.72)$ \\
\hline Performance 3 & 26 & $7.23(1.18)$ & 23 & $6.83(1.59)$ \\
\hline Adherence & 36 & $5.47(1.21)$ & 33 & $5.61(1.50)$ \\
\hline
\end{tabular}

$A M Q$ Athletic Mindfulness Questionnaire, DERS Difficulties in Emotion Regulation Scale

this model had the narrowest CI interval around the parameter estimate. The model showed good fit to data $(\mathrm{PPp}=0.63,95 \%$ confidence interval $=[-8.30,6.10]$. The MAC intervention had a credible effect on training performance $(\beta=-0.10,95 \% \mathrm{CI}=[-0.14,-0.08]$. More specifically, the MAC group reported, on average, higher levels of training performance at T3, compared to the PST group.

\section{Unconditional LGCM}

Dispositional Mindfulness The model showed good fit to the data $(\operatorname{PP} p=0.56,95 \%$ confidence interval $=[-13.54,12.58])$. The athletes reported, on average, a baseline level of $3.49(95 \% \mathrm{CI}=[3.36,3.62])$. During the study, there was a credible increase in dispositional mindfulness $(\beta=0.17,95 \% \mathrm{CI}=[0.12,0.22])$. There were credible variances for both the intercept $\left(\sigma^{2}=0.25,95 \%\right.$ $\mathrm{CI}=[0.16,0.39])$ and the slope $\left(\sigma^{2}=0.02,95 \%\right.$ $\mathrm{CI}=[0.01,0.05])$, which indicates between-person differences in both baseline levels and change of dispositional mindfulness (Fig. 1).
Emotion Regulation The model showed adequate fit to the data $(\operatorname{PP} p=.23,95 \%$ confidence interval $=[-8.35,17.73])$. The athletes reported, on average, a baseline level of 2.53 $(95 \% \mathrm{CI}=[2.359-2.703])$. During the study, there was a credible decrease in emotion regulation difficulties $(\beta=-$ $0.20,95 \% \mathrm{CI}=[-0.27,-0.13])$. There were credible variances for both the intercept $\left(\sigma^{2}=0.39,95 \% \mathrm{CI}=[0.24\right.$, $0.62])$ and the slope $\left(\sigma^{2}=0.02,95 \% \mathrm{CI}=[0.01,0.05]\right)$, which indicates between-person differences in both baseline levels and change of emotion regulation difficulties (Fig. 2).

\section{Mediation Analyses}

Dispositional Mindfulness The models with empirically derived (model A) and less/weakly informative (model C) priors demonstrated adequate data-model fit and the DIC values were also similar for these two models ( $\mathrm{PP} p$ values $\approx 0.20$ ). Additionally, the parameter estimates were in the same direction in all three models with minor differences in magnitude and width of the CI (for estimates, see, Table 3). The hypothesized model A showed less uncertainty regarding the parameter estimates (as indicated by a narrower $\mathrm{CI}$ ) than model $\mathrm{C}$ with less/weakly informative priors on the variances. In light of these results, we chose to focus our presentation and discussion of the results on model A with empirically derived priors.

The selected model (model A) showed adequate fit to data ( $\mathrm{PP} p=0.20,95 \%$ confidence interval $[-10.94,24.38]$. There was a direct effect of the intervention on self-rated training performance $(\beta=-0.11,95 \% \mathrm{CI}=[-0.20,-0.02])$. The participants in the MAC group showed a steeper increase in dispositional mindfulness, in comparison to the PST group $(\beta=$ $-0.58,95 \% \mathrm{CI}=[-0.89,-0.29])$. A steeper increase in dispositional mindfulness was, in turn, related to higher self-rated training performance at $\mathrm{T} 3(\beta=0.03,95 \% \mathrm{CI}=[0.01,0.07])$. The results showed a credible indirect effect of the MAC condition on training performance, measured at T3, through change in dispositional mindfulness $(a b=-0.05,95 \%$ $\mathrm{CI}=[-0.12,-.01])$.

Emotional Regulation The models with empirically derived (model A) and less/weakly informative (model C) priors demonstrated adequate data-model fit ( $\operatorname{PP} p$ values $\approx 0.23$ ). For
Fig. 1 Hypothesized model 1 with standardized parameter estimates. Indirect effect between $\mathrm{MAC}$ and performance via dispositional mindfulness $=$ -0.05 ; *redible association

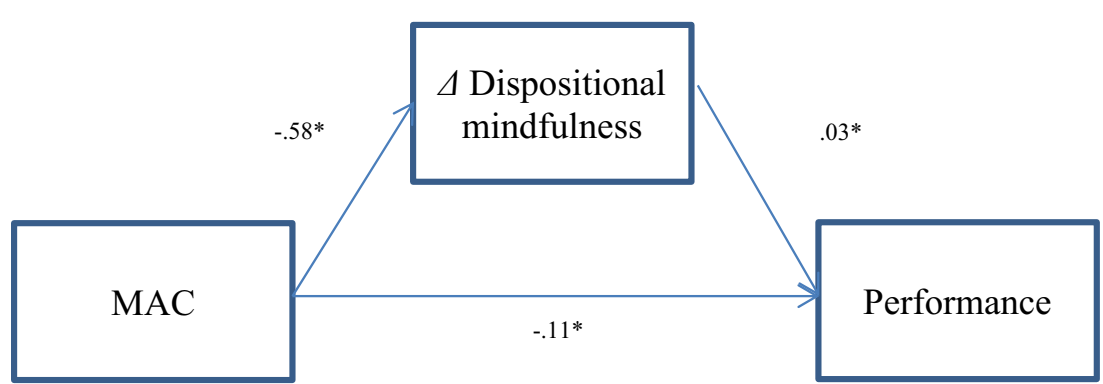


Fig. 2 Hypothesized model 2 with standardized parameter estimates. Indirect effect between $\mathrm{MAC}$ and performance via emotion regulation $=-0.04$ *credible association

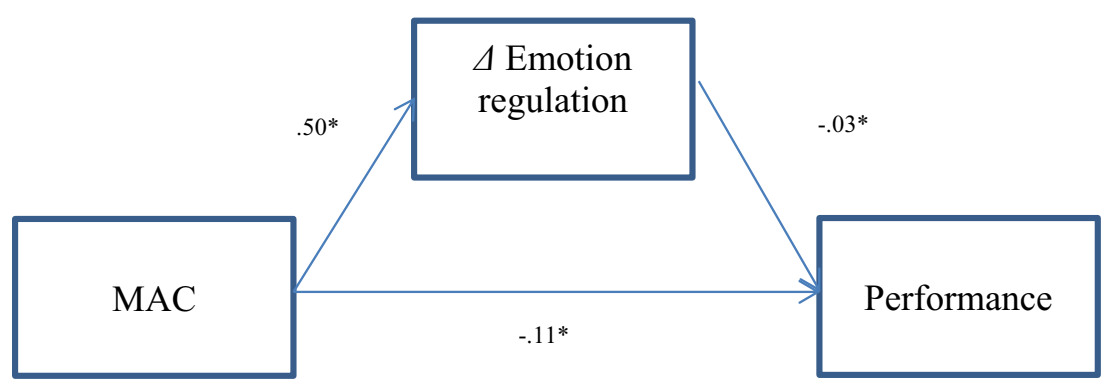

both these models, the DIC values were similar. Additionally, all estimated parameter estimates were in the same direction in all two models with minor differences in magnitude and width of the CI (for estimates, see Table 1). The hypothesized model A showed less uncertainty regarding the parameter estimate (as indicated by a narrower CI) than model $\mathrm{C}$ with a less/weakly informative prior on the variances. In light of these results, we chose to focus our presentation and discussion of the results on the hypothesized model with empirically derived priors.

The selected model showed adequate fit to data ( $\mathrm{PP} p=$ $0.24,95 \%$ confidence interval $[-11.98,23.65])$. There was a direct effect of the intervention on self-rated training performance $(\beta=-0.11,95 \% \mathrm{CI}=[-0.20,-0.01])$. The participants in the MAC group reported a steeper decrease in emotional regulation difficulties, in comparison to the PST group $(\beta=0.50,95 \% \mathrm{CI}=[0.12,0.91])$. A steeper decrease in emotional regulation difficulties was, in turn, related to higher self-rated training performance at T3 $(\beta=-0.03,95 \%$ $\mathrm{CI}=[-0.07,-0.01])$. The results showed a credible indirect effect of the MAC intervention condition on training performance, measured at $\mathrm{T} 3$, through change in emotional regulation $(a b=-0.04,95 \% \mathrm{CI}=[-0.11,-0.01])$.

\section{Discussion}

The general aim of the current study was to explore potential mediating effects of changes in athletic dispositional mindfulness and emotion regulation in the relation between the MAC intervention and self-rated sport performance. Overall, the results support previous theories (e.g., Moore 2009) and suggest that both dispositional athletic mindfulness and emotion regulation appear to be essential mechanisms in the MAC program.

Table 3 Comparisons of parameter estimates using different priors

\begin{tabular}{|c|c|c|c|c|}
\hline & Prior mean (reference) & Model A & Model B & Model C \\
\hline \multicolumn{5}{|l|}{ Hypothesized model 1} \\
\hline MAC-DM & $\begin{array}{l}-0.59 \\
\text { Zhang et al. (2016) }\end{array}$ & $-0.58[-0.89,-0.29]$ & $-0.74[-0.84,-0.64]$ & $-0.47[-0.85,-0.11]$ \\
\hline DM performance & $\begin{array}{l}0.33 \\
\text { Röthlin et al. (2016) }\end{array}$ & $0.03[0.01,0.07]$ & $0.08[0.06,0.11]$ & $0.03[-0.05,0.12]$ \\
\hline MAC performance & $\begin{array}{l}-0.30 \\
\text { Noetel et al. (2017) }\end{array}$ & $-0.11[-0.20,-0.02]$ & $-0.10[-0.13,-0.08]$ & $-0.12[-0.32,0.09]$ \\
\hline Indirect effect ${ }^{\mathrm{a}}$ & $\mathrm{NA}$ & $-0.05[-0.12,-0.01]$ & $-0.17[-0.20,-0.13]$ & $-0.03[-0.17,0.07]$ \\
\hline PPp $[95 \%$ confidence interval $]$ & & $0.20[-10.94,24.38]$ & $0.00[23.92,68.68]$ & $0.22[-10.95,24.17]$ \\
\hline DIC & & 343 & 391 & 344 \\
\hline \multicolumn{5}{|l|}{ Hypothesized model 2} \\
\hline MAC-ER & $\begin{array}{l}0.46 \\
\text { Gross et al. (2017) }\end{array}$ & $0.50[0.12,0.91]$ & $0.74[0.59,0.94]$ & $0.34[-0.16,0.85]$ \\
\hline ER performance & $\begin{array}{l}-0.34 \\
\text { Beedie et al. (2000) }\end{array}$ & $-0.03[-0.07,-0.01]$ & $-0.06[-0.09,-0.04]$ & $-0.03[-0.14,0.04]$ \\
\hline MAC performance & $\begin{array}{l}-0.30 \\
\text { Noetel et al. (2017) }\end{array}$ & $-0.11[-0.20,-0.01]$ & $-0.10[-0.14,-0.08]$ & $-0.12[-0.31,0.09]$ \\
\hline Indirect effect ${ }^{\mathrm{a}}$ & NA & $-0.04[-0.11,-0.01]$ & $-0.13[-0.17,0.10]$ & $-0.03[-0.16,0.05]$ \\
\hline PPp [95\% confidence interval] & & $0.24[-11.98,23.65]$ & $0.01[8.61,47.95]$ & $0.25[-12.94,21.90]$ \\
\hline DIC & & 403 & 429 & 403 \\
\hline
\end{tabular}

${ }^{a}$ For the indirect effects, the unstandardized estimates are presented. Model A, hypothesized model with empirical priors for parameter estimates and variances at 0.02 ; model B, empirical priors for the parameter estimates and highly precise priors were sent for the parameter estimates variances (i.e., 0.001); model C, empirical priors for the parameter estimates and low precise priors for the parameter estimates variances (i.e., 0.2 ) 


\section{Dispositional Mindfulness}

As expected, the MAC group obtained greater improvements in dispositional sport-specific mindfulness skills and training performance compared to the PST group. This result is partly similar to findings in previous studies (i.e., Goodman et al. 2014; Gross et al. 2016; Hasker 2010). However, the results in the current study are not entirely comparable to earlier studies, considering that Goodman et al. (2014) did not use an active control group and tested MAC in combination with hatha yoga, and further, that the aforementioned MAC studies (Goodman et al. 2014; Gross et al. 2016; Hasker 2010) used "general" mindfulness questionnaires to assess dispositional mindfulness, whereas we used a mindfulness measure that was specifically designed for athletes.

Still, what MAC and PST do have in common is that both interventions try to increase focus on current sport-relevant tasks but they differ considerably in how this should be done. The present results suggest that increases in athletic mindfulness skills (i.e., contextual awareness, present-moment attention to task-relevant stimuli, and acceptance) may be stronger and more fundamental in MAC compared to traditional PST techniques. Improving attention and awareness skills on present-moment experiences may be crucial in order to gain a deeper insight into behavioral, cognitive, and emotional patterns the athlete thereafter can apply acceptance skills to. Consequently, athletes who cultivate both attention and acceptance may gradually become more and more aware of their mental and emotional processes, and they also may become quicker to notice them (Josefsson et al. 2017; Lindsay and Creswell 2017).

The particular combination of increases in attention/ awareness and acceptance may result in overall improvements in adaptive behaviors (Chambers et al. 2009) that in turn may be particularly important for athletes who are expected to perform optimally, sometimes under very challenging and stressful conditions (Birrer and Morgan 2010). Improvements in sustained attention capacities during training and competition may make it easier for athletes to stay focused on current taskrelevant stimuli, and make them less vulnerable to various distractions that may inhibit high-level performance (Gardner and Moore 2012; Marks 2008). Also, enhanced attention abilities may increase athletes' capacities to read the game and seize offensive and defensive opportunities. For example, in team sports (e.g., floorball, soccer, ice hockey) rapid changes can suddenly lead to unexpected openings and breakthroughs that may result in scoring opportunities that a less attentive mind might miss. Similarly, an increased overall self-awareness, combined with greater acceptance of mental and emotional content, may also contribute to enhanced focus on the relevant task at hand, and promote self-regulated values-directed behaviors and decrease avoidance and safety behaviors (Giges and Reid 2016).
The defusing and accepting processing of thoughts and emotions, taught and practiced in MAC, may provide a more economical way to use cognitive resources compared to a PST control-based approach because no conscious efforts are made to suppress or inhibit disturbing mental and emotional content (Gardner and Moore 2012; Marks 2008). If less attention is paid on thoughts and feelings, then greater attentional resources are available for sport tasks (Moore 2016). Hence, it may cognitively "cost" more to try and get rid of unwanted internal experiences than to just mindfully accept them and "let them be" (Gardner and Moore 2012).

In line with the view presented by Borkovec (2002), the mindful athlete, who manages to focus on external current task-relevant stimuli and yet is able to maintain contextual awareness, may optimally process present information and, by doing so, increase the possibility that the athlete makes the right decision in current challenging situations, despite the rapid changes that often occur in team sports. An enhanced ability to keep focus on external stimuli may be particularly relevant for athletes considering that self-focused attention on automatized movements is associated with performance decreases (Masters and Maxwell 2008). With the addition of acceptance to enhanced awareness and attention skills, a "mindful mind" may provide the ideal preconditions for peak sport performance (Josefsson et al. 2017).

The acceptance feature in MAC may lead not only to an increased overall acceptance of the self but also to an improved mindful acceptance of disturbing thoughts and feelings. If athletes can accept themselves as they are, then it may be easier to also accept unpleasant thoughts and feelings as well as unexpected and unwanted events during training or competition. Increased acceptance of mistakes made during matches and competitions could make it easier for athletes to let go of judgements and self-denigrating thoughts and their accompanying negative emotions (Carson and Langer 2006). Additionally, acceptance may also reduce fear of failure that, in turn, may help athletes feel courageous enough to dare to try innovative and creative solutions to various problematic situations during their matches (Carson and Langer 2006).

\section{Emotion Regulation}

The current study showed that emotion regulation difficulties decrease following the MAC intervention. These decreases are also correlated with increases in participants' selfassessed training performance, which may be related to improved competitive performance. These findings are consistent with both previous research (Gross et al. 2016) and theories stating that emotion regulation is a core mechanism of 
action in the MAC program (e.g., Moore 2016). Increased emotional awareness after a MAC-based treatment may help athletes quickly detect emerging disruptive emotions that, if left unregulated, could lead to maladaptive cognitive and behavioral responses, which might negatively influence athletic performance (Josefsson et al. 2017). Moreover, strong affects such as fear may also reduce motivation and self-confidence, as well as enhance worry and anxiety in competitive athletes (Birrer and Morgan 2010).

An increasing ability to accept all emotions and affective states may prevent athletes from getting caught up in potentially performance-inhibiting strong emotions (Moore 2016). Moreover, the acceptance element in MAC may help athletes reduce experiential avoidance as well as increase their tolerance of unpleasant emotional states, which may make it easier for athletes to pursue values-directed behaviors, as opposed to emotion-driven avoidant behaviors (Moore 2016). Disturbing emotional states may often emerge when things do not work out as planned in a competition and a defeat seems inevitable (Josefsson et al. 2017). Increased acceptance, as well as the defusion aspect taught in MAC, may help athletes sit with distressing emotions and thoughts that disappointing defeats often trigger.

One may think that emotion regulation only is relevant when it comes to threatening and distressing emotions but ego-boosting emotions such as pride and various types of self-celebratory feelings of being invincible may not be helpful for athletes because they also tend to disrupt focus on the present external sport-relevant task. Likewise, positive feelings of joy and relief that arise when training or competition performance has been successful may also interrupt external attention to the present task. For example, Baumeister and Steinhilber (1984) found that choking effects also can occur in situations when a team is on the verge of winning an important match. From a MAC perspective, athletes need to attend to external task-relevant present stimuli and mindfully accepting whatever internal stimuli, such as negative emotions, that may emerge in the present moment, rather than trying to alter or reduce them (Gardner and Moore 2012).

\section{Limitations}

There are several limitations of the present study that need to be recognized. First, considering that the population mainly consisted of floorball players and golfers, the generalizability to other sports are unclear. Second, the use of self-reported measures is a potential limitation for the study because it is likely that the results, to some extent, are influenced by common method biases (for a discussion about common method biases, see Podsakoff et al. 2012). To decrease the potential influence of common method biases (CMBs), we followed the recommendations from Podsakoff et al. (2012), by clearly explaining the motive for conducting the study and how the information they provided would be used. A longitudinal design also decreases potential CMB. Further, even if previous researchers have called for objective performance measures when examining the effectiveness of psychological interventions aimed at enhancing athletic performance (e.g., Martin et al. 2005), we deemed it impossible to develop a general objective performance measure that would be sufficiently adequate for a multiple-sport sample, consisting of both individual- and team-sport athletes.

Moreover, the performance item was developed specifically for this study and the psychometric properties of the item have not been established. We also made the decision to only assess training performance because there was a great variance in number of matches/competitions during the intervention, and the intervals between the matches/competitions also differed to a great extent among the participants. It would be desirable to use objective performance measures in future trials. In team sports, it is, however, more or less impossible to objectively assess performance because the players are engaged in numerous numbers of situations during a match and aside from scoring goals and goal assists, there are no scores or times that can be evaluated. Moreover, different players have different roles and different tasks which make it even more complicated to evaluate each player's performance objectively.

Further, even if mindfulness questionnaires often show good psychometric properties (Grossman and Van Dam 2011), it should be acknowledged that these questionnaires have been criticized for several reasons, among other things that the understanding of keywords frequently used in measures (e.g., "paying attention," "awareness," "acceptance," "judging") is dependent on previous meditation experience and/or familiarity with mindfulness practice, and, also, that the concordance between how mindful a person thinks he/ she is and his/her true level of mindfulness may not necessarily be sufficiently accurate (Grossman and Van Dam 2011).

Finally, data of practice in between-sessions were not collected. Thus, we do not know to what extent participants engaged in mindfulness exercises at home. Some athletes may have been more interested and motivated to practice mindfulness while others may not have practiced at all.

RCTs examining the effectiveness of MAC on performance- and process-relevant variables are warranted, using both subjective and objective performance measures. MAC-related effects on facets of dispositional mindfulness should specifically be examined. In addition, we suggest that the athletes should be clinically classified, using the MCS-SP, which makes it possible to study potential moderating effects of clinical level. MAC effects on performance may unfold gradually over time. For that reason, long-term MAC effects should also be examined. Future longitudinal studies, using a mixed-method design, should also try to identify which aspects of performance-relevant behaviors are particularly influenced by MAC. 
Author Contributions TJ designed and executed the study, assisted with the data analyses, and wrote the paper. AI collaborated with the design and the writing of the manuscript as well as analyzed the data. HG and ML collaborated with the writing of the paper. AS assisted with data analyses and writing of the results. RT and JB collaborated in the data collections. All authors critically reviewed the manuscript prior to submission.

Funding This research project was funded by grants from the Swedish Research Council for Sport Science (D2016-0037/P2016-0146) and from the Center of Research on Welfare, Health, and Sport, Halmstad University, Sweden.

Compliance with Ethical Standards All procedures performed in studies involving human participants were in accordance with the ethical standards of the institutional and/or national research committee and with the 1964 Helsinki declaration and its later amendments or comparable ethical standards. Informed consent was obtained from all individual participants included in the study. The study was reviewed and approved by the regional ethical review board of Lund University (no. 2016/153).

Conflict of Interest The authors declare that they have no conflict of interest.

Open Access This article is distributed under the terms of the Creative Commons Attribution 4.0 International License (http:// creativecommons.org/licenses/by/4.0/), which permits unrestricted use, distribution, and reproduction in any medium, provided you give appropriate credit to the original author(s) and the source, provide a link to the Creative Commons license, and indicate if changes were made.

Publisher's Note Springer Nature remains neutral with regard to jurisdictional claims in published maps and institutional affiliations.

\section{References}

Bandura, A. (1997). Self-efficacy: The exercise of control. New York: WH Freeman/Times Books/Henry Holt \& Co.

Baskin, T. W., Tierney, S. C., Minami, T., \& Wampold, B. E. (2003). Establishing specificity in psychotherapy: a meta-analysis of structural equivalence of placebo controls. Journal of Consulting and Clinical Psychology, 71, 973-979.

Baumeister, R. F., \& Steinhilber, A. (1984). Paradoxical effects of supportive audiences on performance under pressure: the home field disadvantage in sports championships. Journal of Personality and Social Psychology, 47, 85-93.

Beedie, C. J., Terry, P. C., \& Lane, A. M. (2000). The profile of mood states and athletic performance: two meta-analyses. Journal of Applied Sport Psychology, 12, 49-68.

Birrer, D., \& Morgan, G. (2010). Psychological skills training as a way to enhance an athlete's performance in high-intensity sports. Scandinavian Journal of Medicine \& Science in Sports, 20, 78-87.

Birrer, D., Röthlin, P., \& Morgan, G. (2012). Mindfulness to enhance athletic performance: theoretical considerations and possible impact mechanisms. Mindfulness, 3, 325-246.

Borkovec, T. D. (2002). Life in the future versus life in the present. Clinical Psychology: Science and Practice, 9, 76-80.

Brefczynski-Lewis, J. A., Lutz, A., Schaefer, H. S., Levinson, D. B., \& Davidson, R. J. (2007). Neural correlates of attentional expertise in long-term meditation practitioners. PNAS, 104, 11483-11488.

Bühlmayer, L., Birrer, D., Röthlin, P., Faude, O., \& Donath, L. (2017). Effects of mindfulness practice on performance-relevant parameters and performance outcomes in sports: a meta-analytical review. Sports Medicine. https://doi.org/10.10007/s40279-0170752-9.

Cahn, B. R., \& Polich, J. (2009). Meditation (Vipassana) and the P3a event-related brain potential. International Journal of Psychophysiology, 72, 51-60.

Carron, A. V., \& Hausenblas, H. A. (1998). Group dynamics in sport (2nd ed.). Morgantown: Fitness Information Technology.

Carson, S. H., \& Langer, E. J. (2006). Mindfulness and self-acceptance. Journal of Rational-Emotive \& Cognitive-Behavior Therapy, 24, 29-43. https://doi.org/10.1007/s10942-006-0022-5.

Chambers, R., Gullone, E., \& Allen, N. B. (2009). Mindful emotion regulation: an integrative review. Clinical Psychology Review, 29, $560-572$.

Coffey, K. A., Hartman, M., \& Fredrickson, B. L. (2010). Deconstructing mindfulness and constructing mental health: understanding mindfulness and its mechanisms of action. Mindfulness, 1, 235-253.

Deci, E., \& Ryan, R. (2000). The what and why of goal pursuits: human need and the self-determination of behavior. Psychological Inquiry, $11,227-268$.

Eifert, G. H., \& Forsyth, J. P. (2005). Acceptance and commitment therapy for anxiety disorders. Oakland: New Harbinger.

Elliot, A. J., Murayama, K., \& Pekrun, R. (2011). A 3x2 achievement goal model. Journal of Education Psychology, 103, 632-648. https://doi.org/10.1037/a0023952.

Gardner, F. L. (2009). Efficacy, mechanisms of change, and the scientific development of sport psychology. Journal of Clinical Sport Psychology, 3, 139-155.

Gardner, F. L., \& Moore, Z. E. (2004). A mindfulness-acceptancecommitment (MAC) based approach to athletic performance enhancement: Theoretical considerations. Behavior Therapy, 35, 707-723.

Gardner, F. L., \& Moore, Z. E. (2007). The psychology of enhancing human performance: the mindfulness-acceptance-commitment approach. New York: Springer.

Gardner, F. L., \& Moore, Z. E. (2012). Mindfulness and acceptance models in sport psychology: a decade of basic and applied scientific advancements. Canadian Psychology, 53, 309-318.

Giges, B., \& Reid, G. (2016). Awareness, self-awareness, and mindfulness: the application of theory to practice. In A. L. Baltzell (Ed.), Mindfulness and performance. Current perspectives in social and behavioral sciences (pp. 488-512). Cambridge University Press.

Goodman, F. R., Kashdan, T. B., Mallard, T. T., \& Schumann, M. (2014). A brief mindfulness and yoga intervention with an entire NCAA divison 1 athletic team: an initial investigation. Psychology of Consciousness: Theory, Research, and Practice, 1, 339-356. https://doi.org/10.1037/cns0000022.

Gratz, K. L., \& Roemer, L. (2004). Multidimensional assessment of emotion regulation and dysregulation: development, factor structure, and initial validation of the Difficulties in Emotion Regulation Scale. Journal of Psychopathology and Behavioral Assessment, 26, 41-54.

Gratz, K. L., \& Tull, M. T. (2010). Emotion regulation as a mechanism of change in acceptance-and mindfulness-based treatments. In R. A. Baer (Ed.), Assessing mindfulness and acceptance processes in clients: Illuminating the theory and practice of change (pp. 107-133). Oakland: New Harbinger Publications.

Gross, J. J. (1998). The emerging field of emotion regulation: an integrative review. Review of General Psychology, 2, 271-299.

Gross, M., Moore, Z. E., Gardner, F. L., Wolanin, A. T., Pess, R., \& Marks, D. R. (2016). An empirical examination comparing the mindfulness-acceptance-commitment approach and psychological skills training for the mental health and sport performance of female student athletes. International Journal of Sport and Exercise Psychology. https://doi.org/10.1080/1612197X.2016.1250802.

Grossman, P., \& Van Dam, N. T. (2011). Mindfulness, by any other name...: trials and tribulations of Sati in Western psychology and science. Contemporary Buddhism, 12, 219-239. 
Gustafsson, H., Lundqvist, C., \& Tod, D. (2017). Cognitive behavioral intervention in sport psychology: a case illustration of the exposure method with an elite athlete. Journal of Sport Psychology in Action, 8(3), 152-162. https://doi.org/10.1080/21520704.2016.1235649.

Harge, O. (2006). The handbook of communication skills. Hove: Routledge.

Hasker, S. M. (2010). Evaluation of the mindfulness-acceptance-commitment (MAC) approach for enhancing athletic performance (Doctoral dissertation). Indiana, PA: Indiana University of Pennsylvania.

Hayes, S. C., \& Feldman, G. (2004). Clarifying the construct of mindfulness in the context of emotion regulation and the process of change in therapy. Clinical Psychology: Science and Practice, 11, 255-262. https://doi.org/10.1093/clipsy/bph080.

Hayes, S. C., Strosahl, K. D., \& Wilson, K. G. (1999). Acceptance and commitment therapy: an experiential approach to behaviour change. New York: Guildford.

Ivarsson, A., Andersen, M., Stenling, A., Johnson, U., \& Lindwall, M. (2015). Things we haven't learned (so far). Journal of Sport \& Exercise Psychology, 37, 449-461.

Josefsson, T., Ivarsson, A., Lindwall, M., Gustafsson, H., Stenling, A., et al. (2017). Mindfulness mechanisms in sports: mediating effects of rumination and emotion regulation on sport-specific coping. Mindfulness. https://doi.org/10.1007/s12671-017-0711-4.

Kaplan, D., \& Depaoli, S. (2012). Bayesian structural equation modeling. In R. Hoyle (Ed.) Handbook of structural equation modeling (pp. 650-673). New York: Guilford Press.

Khoury, B., Lecomte, T., Fortin, G., Masse, M., Therien, P., Boluchard, V., Chapleau, M.-A., Paquin, K., \& Hofmann, S. G. (2013). Mindfulness-based therapy: a comprehensive meta-analysis. Clinical Psychology Review, 33, 763-771.

Lane, A. M., \& Chappell, R. H. (2001). Mood and performance relationships at the world student games basketball competition. Journal of Sport Behavior, 24, 182-196.

Lindsay, E. K., \& Creswell, J. D. (2017). Mechanisms of mindfulness training: Monitor and Acceptance Theory (MAT). Clinical Psychology Review, 51, 48-59. https://doi.org/10.1016/j.cpr.2016.10.011.

Lowther, J., Lane, A. M., \& Lane, H. (2002). Self-efficacy and psychological skills during the amputee soccer world cup. Athletic Insight, 4, 23-34.

Lutz, A., Greischar, L. L., Rawlings, N. B., Ricard, M., \& Davidson, R. J. (2004). Long-term meditators self-induce high-amplitude gamma synchrony during mental practise. PNAS, 101, 16369-16373.

MacCoon, D. G., Imel, Z. E., Rosenkranz, M. A., Sheftel, J. G., Weng, H. Y., Sullivan, J. C., et al. (2012). The validation of an active control intervention for Mindfulness Based Stress Reduction (MBSR). Behaviour Research and Therapy, 50, 3-12. https://doi.org/10. 1016/j.brat.2011.10.011.

Marks, D. R. (2008). The Buddha's extra scoop: neural correlates of mindfulness and clinical sport psychology. Journal of Clinical Sport Psychology, 2, 216-241.

Martin, G. L., Vause, T., \& Schwartzman, L. (2005). Experimental studies of psychological interventions with athletes in competition. Why so few? Behavior Modification, 29, 616-641. https://doi.org/10.1177/ 0145445503259394.

Masters, R., \& Maxwell, J. (2008). The theory of reinvestment. International Review of Sport and Exercise Psychology, 2, 160-183.

Mohr, D. C., Spring, B., Freedland, K. E., Beckner, V., Arean, P., Hollon, J., \& Kaplan, R. (2009). The selection and design of control conditions for randomized controlled trials of psychological interventions. Psychotherapy and Psychosomatics, 78, 275-284. https://doi.org/ $10.1159 / 000228248$.

Moore, Z. E. (2009). Theoretical and empirical developments of the mindfulness-acceptance-commitment (MAC) approach to performance enhancement. Journal of Clinical Sports Psychology, 4, 291-302.

Moore, Z. E. (2016). Mindfulness, emotion regulation, and performance. In A. L. Baltzell (Ed.), Mindfulness and performance, current perspectives in social and behavioral sciences (pp. 29-52). Cambridge University Press.

Muthén, B., \& Asparouhov, T. (2012). Bayesian structural equation modeling: A more flexible representation of substantive theory. Psychological Methods, 17, 317-335.

Noetel, M., Ciarocchi, J., Van Zanden, B., \& Lonsdale, C. (2017). Mindfulness and acceptance approaches to sporting performance enhancement: a systematic review. International Review of Sport and Exercise Psychology. https://doi.org/10.1080/1750984X.2017.1387803.

Pagnoni, G., \& Cekic, M. (2007). Age effects on gray matter volume and attentional performance in Zen meditation. Neurobiology of Aging, $28,1623-1627$.

Podsakoff, P. M., MacKenzie, S. B., \& Podsakoff, N. P. (2012). Sources of method bias in social science research and recommendations on how to control it. Annual Review of Psychology, 63, 539-569. https://doi.org/10.1146/annurev-psych-120710-100452.

Ritschel, L. A., Tone, E. B., Schoemann, A. M., \& Lim, N. E. (2015). Psychometric properties of the Difficulties in Emotion Regulation Scale across demographic groups. Psychological Assessment, 27, 944-954. https://doi.org/10.1037/pas0000099.

Roemer, L., Williston, S. K., \& Rollins, L. G. (2015). Mindfulness and emotion regulation. Current Opinion in Psychology, 3, 52-57.

Roesch, S., Norman, G. J., Vollodas, F., Sallis, J. F., \& Patrick, K. (2010). Intervention-mediated effects for adult physical activity: a latent growth curve analysis. Social Science \& Medicine, 71, 494-501.

Röthlin, P., Horvath, S., Birrer, D., \& Holtforth, M. (2016). Mindfulness promotes the ability to deliver performance in highly demanding situations. Mindfulness, 7, 727-733.

Schultz, K. F., \& Grimes, D. A. (2002). Generation of allocation sequences in randomised trials: chance not choice. The Lancet, 359, 515-519.

Smith, H. W. (1994). The 10 natural laws of successful time and life management: proven strategies for increased productivity and inner peace. New York: Warner.

Song, X. Y., \& Lee, S. Y. (2012). Basic and advanced Bayesian structural equation modeling: With applications in the medical and behavioral sciences. Hoboken, NJ: Wiley. https://doi.org/10.1002/ 9781118358887.

Stenling, A., Ivarsson, A., \& Lindwall, M. (2015). The only constant is change: Analysing and understanding change in sport and exercise psychology research. International Review of Sport and Exercise Psychology, 10, 230-251.

Van de Schoot, R., Kaplan, D., Denissen, J., Asendorpf, J. B., Neyer, F. J., \& van Aken, M. A. (2014). A gentle introduction to Bayesian analysis: applications to developmental research. Child Development, 85(3), 842-860.

Wegner, D. M. (1989). Ironic processes of mental control. Psychological Review, 101, 34-52.

Wenzlaff, R. M., \& Wegner, D. M. (2000). Thought suppression. Annual Review of Psychology, 51, 59-91.

Youngblut, J. M., Casper, G. R., \& Bolton, F. P. (2013). Focus on psychometrics single-item indicators in nursing research. Nursing Research \& Health, 16, 459-465.

Yuan, Y., \& MacKinnon, D. P. (2009). Bayesian mediation analysis. Psychological Methods, 14, 301-322.

Zhang, C.-Q., Chung, P.-K., \& Si, G. (2015). Assessing acceptance in mindfulness with direct-worded items: the development and initial validation of the athlete mindfulness questionnaire. Journal of Sport and Health Science, 6, 311-320. https://doi.org/10.1016/j.jshs.2015.09.010.

Zhang, C.-Q., Si, G., Duan, Y., Lyu, Y., Keatley, D. A., \& Chan, D. K. C. (2016). The effects of mindfulness training on beginners' skill acquisition in dart throwing: a randomized controlled trial. Psychology of Sport and Exercise, 22, 279-285. https://doi.org/10.1016/j. psychsport.2015.09.005.

Zyphur, M. J., \& Oswald, F. L. (2015). Bayesian estimation and inference: a user's guide. Journal of Management, 41, 390-420. 